\title{
Nurses are Vital to Improving Clinical Integrity and Documentation in Hospitals
}

\author{
Everett-Thomas $\mathrm{R}^{*}$, Brito Y and Joseph L \\ University of Miami School of Nursing and Health Studies, United States
}

${ }^{*}$ Corresponding author: Ruth Everett-Thomas, Assistant Professor of Clinicals, University of Miami School of Nursing and Health Studies, 5030 Brunson Drive suite, Coral Gables, Florida, United States, Tel: 305 284-3666, E-mail: reverett@med.miami.edu

Citation: Everett-Thomas R, Brito Y, Joseph L (2018) Nurses are Vital to Improving Clinical Integrity and Documentation in Hospitals. J Nurs Healthcare Manag 1: 104

Article history: Received: 07 February 2018, Accepted: 22 June 2018, Published: 23 June 2018

\begin{abstract}
Background: Nurses play a vital role as clinical documentation improvement (CDI) specialists to facilitate the coordination of patient care by providing a concurrent review of medical records for every hospitalized patient. They serve as liaisons between physicians and medical coders to ensure accurate coding, improve reimbursements, patient safety and outcomes. CDI specialist may provide support to a more transparent healthcare team by making recommendations for care based upon patient findings after thorough investigation of all laboratory and diagnostic tests along with the physicians' narrative.

Method: This paper used two case studies to describe the role of a nurse CDI specialist in a hospital setting. Information from the case studies were used to show the importance of a collaboration between a nurse CDI specialist and physician to improve patient care and provide accurate coding.

Discussion/Conclusion: CDI specialist provide additional oversight to help prevent billing and coding mistakes that may lead to federal investigations and or poor patient outcomes. Nurses may be optimal CDI specialists because their education and clinical experience helps them evaluate many aspects of the medical record to make recommendations for care based upon best evidence-based practice. They are able to understand the patients diagnoses and ensure that the patient's course of hospitalization is aligned with accurate treatment. Thus, providing a bridge between clinical coders and physicians by interpreting and coding narrative data concurrently so reimbursements are accurate when completed post discharge.
\end{abstract}

Keywords: Nurses Role; Clinical Integrity; Documentation; Patient Outcomes; Patient Safety

\section{Introduction}

The United States has the world's highest per capita health care costs, and tax-funded expenditures accounted for nearly $64.3 \%$ of US health care spending [1]. Although hospital associated costs continue to rise, the US government sought a way to control costs while encouraging hospitals to provide care more efficiently [2]. Starting in the 1980's, The Centers for Medicare and Medicaid Services, developed a coding system using Diagnosis-Related Group (DRG) to provide guidelines for reimbursement of funds to physicians and medical organizations for services rendered [2]. Diagnosis-Related Group (DRG) is a statistical system of classifying any inpatient stay into groups for the purposes of payment [2]. Rather than paying the hospital for what it spent caring for a hospitalized patient, Medicare pays the hospital a fixed amount based on the patient's DRG or diagnosis [2].

In an effort to improve the federal reimbursement system and broaden the codes related to patient diagnoses, The Centers for Medicare and Medicaid Services implemented the International Classification of Diseases (ICD) system developed by the World Health Organization as a way of capturing many comorbidities [3]. Though revising the coding guidelines to meet evidence-based practice standards was necessary, they also created challenges to accurately code services that provide care more efficiently. This process has proven to be time consuming across many health care settings, and increased the awareness of fundamental coding rules, guidance and governance [4]. Subsequently requiring an increase number of medical billing and coding professionals to examine large volumes of data that would facilitate medical record management [5]. Most clinical coders are certified in medical billing and help by promoting consistent, precise, reliable, non-contradictory documentation integral to the compliant submission 
of Health Insurance Portability and Accountability Act transaction (code) sets [4]. Clinical coders must take the physicians narrative notes and determine what additional documentation is needed for accurate coding and reporting required by the government as defined by various healthcare code sets [6,7]. They look for key words in the physician narrative notes to assign a code that depicts the clinical diagnoses. This process is performed after patient discharge to guarantee adequate reimbursement for the hospital and provider [5].

With the complexity of disease conditions and advanced treatment modalities, coders may need additional support in understanding the patient's course of hospitalization [8]. Nurses could provide that additional support because of their direct influence on patient outcomes. They can bridge the gap between coders and physicians in the role of clinical documentation improvement (CDI) specialists using their experience and education to ensure that patient care is aligned with appropriate diagnoses and treatments [9]. CDI specialists are individuals that review patient records concurrently to ensure that medical data is aligned with the diagnoses and treatments the patient received in a healthcare setting [10]. CDI specialist review medical records in the patient care area and are able to discuss discrepancies in patient diagnoses and treatments with the physician. This clarification of diagnoses in real-time allows the physician and CDI specialist to build a collaboration which may include other members of the health care team [8]. These specialists are trained and certified in data collection and interpretation for organizations to obtain payment for services rendered, improve patient outcomes, reduce hospital days, and prevent fraudulent billing [10]. CDI specialists act as mediators between billing/coding personnel who translate diagnoses into data, and healthcare providers that offer direct patient care [11]. This paper will discuss the role of a nurse as a CDI specialist in providing additional oversight for patent safety through data investigation described in two case studies. Suggesting that the collaboration between the CDI specialist and physician to clarify patient care discrepancies in real-time may be more beneficial to the patient, provider and hospital.

\section{Patient Safety}

The Agency for Healthcare Research and Quality (AHRQ) developed patient safety indicators to provide guidance regarding the relevant documentation and coding process. This process uses narrative descriptions of diseases, injuries, and health care procedures and transform them into numeric or alphanumeric designations (code numbers) [14]. Code numbers are detailed to accurately describe the diagnoses and the procedures performed to test or treat these diagnoses. Therefore, accurate quality measurement and reporting must now focus on coding for appropriate reimbursement and patient safety indicators. To establish the codes for each inpatient stay, coders must be able to decipher the narrative documentation provided by physicians and other healthcare workers [15].

Knowledge and experience of the CDI specialists are needed to help provide a consistent code language using specific diagnostic terms. These specialists are also liaisons between documentation and care by providing a process to request additional information from physicians regarding questions or issues with treatment modalities [11,12]. Subsequently, nurses' education and experience are key components for CDI specialists to ensure that patients receive appropriate care, and healthcare organizations are reimbursed for services rendered. The following two cases will describe a simplified version of a CDI specialist task in providing data integrity to the documentation process. Each case study consist of true patient information using limited data to prevent patient identification while providing a sequential course. Also, the cases below were investigated by a registered nurse certified CDI specialist with a master's degree in nursing education and more than 10 years of clinical experience.

\section{Methods}

A senior clinical nurse researcher collaborated with a nurse CDI specialist to describe the role and value of clinical integrity and documentation in one large academic hospital. The 560-bed teaching hospital is in a downtown metropolitan area and is one of three health care facilities serving individuals with private and public insurance. Local universities and other academic institutions use these facilities to train health care professionals in many different specialties. The CDI specialist program was one of latest courses added to train nurses to become liaisons between physicians and coders to clarify at-risk documentation prior to claim submission. This course used the American Health Information Management Association 2014 Clinical Documentation Toolkit to guide the program's implementation [11].

In this hospital, the CDI specialist perform a concurrent review of the patient's medical record as a way to increase accuracy, clarity, and specificity of provider documentation [12]. The CDI specialist also ensure that federal codes for billing are accurate and patients are receiving the appropriate treatment based upon their diagnoses. Along with the daily reviewing of medical records, CDI specialists use evidence-based data to confirm that correct medical treatment is provided to patients based upon current recommendations from experts [13]. These specialists then meet with providers to discuss their findings and suggest recommendations to attest that patients receive the appropriate treatment for optimal outcomes and improved patient safety [11].

\section{Findings}

\section{Case Study \# 1}

Patient \#1 was admitted to the hospital via the emergency department with a chief complaint of anemia, (unspecified coding DRG 812) accompanied with dizziness and weakness. After a physical exam and routine lab work was completed prior to admission, the 
clinical findings were listed as unable to determine the cause of bleeding. However, the physician's narrative notes suggests that the bleeding could be related to a previous diagnosis of liver cirrhosis. Additional labs were drawn and the results corroborated with the active bleeding diagnoses presenting a hemoglobin change from 9.6 to 7.6. The admitting physician recommended two units of blood transfusion if the hemoglobin dropped below 7.0 and continued monitoring of the patient's condition.

After review of the medical record by the CDI specialist and meeting with a physician, a diagnosis clarification was requested for gastrointestinal hemorrhage (coding DRG 378T) since the patient previous medical history from another hospitalization included; cholecystectomy, variceal banding, thoracentesis $\mathrm{x} 2$, and cirrhosis. The CDI specialist recommended that the medical team evaluate the patient's gastrointestinal system to identify the location of the bleeding and treat accordingly. Although the CDI specialist and physician caring for the patient agreed that two units of blood is a necessary treatment for anemia due to blood loss, however, treatment alone does not provide care more efficiently. This patient received two units of blood and remained in the hospital until a variceal banding procedure was performed. Additional tests were done to ensure that the patient hemoglobin levels were back into the normal range and the patient no longer displayed signs and symptoms of anemia. The CDI specialist continued to provide a concurrent review of the medical records to ensure that appropriate care was aligned with the patient diagnosis.

In the above scenario, it is presumed that this patient could have slipped through the cracks if the medical record was reviewed after discharge and the patient was only given two units of blood. Although the coding and treatment for anemia would have been correct, in hindsight this post review of the medical record could have resulted in additional treatment or prolonged suffering and or even death. The CDI specialists were able to review the patient's record and meet with the treating physician to make a recommendation for a diagnosis based upon the patient's admitting information, previous medical history, and pathophysiology for complications related to liver cirrhosis. These specialists provide additional oversight to improve patient safety and prevent billing fraud through exhaustive investigation of the medical record for each patient hospitalization.

\section{Case Study \#2}

Patient \#2 was admitted to the hospital with a diagnosis of Pancreatic Cancer (Malignant neoplasm of pancreas-unspecified DRG-435), and the patient's conditioned worsened over a two-week period as the patient became confused with an elevated blood pressure. All other vital signs for this patient were in normal range. This patient had an indwelling central venous catheter and a positive blood culture with gram negative rods. The team assumed the infection was due to the indwelling catheter and a new diagnosis of bacteremia (DRG-286) was assigned. Removal of the catheter was first priority and the patient received broadspectrum antibiotics. However, over the next few days the patient remained confused and subsequent microbiology results from the indwelling catheter were negative.

After further investigation by the CDI specialist, a recommendation was made to associate the infection to secondary biliary outlet obstruction which is a known risk factor for pancreatic head tumor versus catheter- associated blood stream infection. Additional laboratory values for this patient were normal including hemoglobin, hematocrit, and platelet count. Consequently, suggesting that the infection was not related to the indwelling catheter and the changes in vital signs could have been associated with the patient's past medical history of hypertension and diabetes. In this scenario, the medical team assumed that the worsening of the patient's medical condition was related to one of the most common causes of blood stream infections (catheter associated), however, the patient did not present with the typical signs and symptoms related to bacteremia. The CDI specialists used their experience and the pathophysiology of the patient's disease to help determine that the infection may have been related to the patient's disease process and not the indwelling catheter. Thus, the CDI specialist reviewing the patient's record concurrently, provided additional information for the physician as a recommendation to deliver more efficient care. In both case studies, the CDI specialists helped improve patient outcomes through concurrently reviewing the patient's medical records during course of hospitalization. A second layer of oversight also provides accurate coding to ensure that reimbursement of services is aligned with the care received and prevent fraudulent billing due to coding errors.

\section{Discussion}

While healthcare fraud is a serious crime, it is not limited to any one professional group. In 2016, the US Department of Justice (DOJ) arrested more than 300 individuals that included physicians, nurses, pharmacists, and other healthcare professionals on charges related to conspiracy to commit health care fraud. These charges included, violations of the anti-kickback statutes, money laundering, aggravated identity theft, and services not rendered or related to home health care and prescription drugs [16]. The US Department of Justice reported that $\$ 900$ million dollars were lost in healthcare funds due to fraudulent billing. In this report, most of the billing claims were done with an intention to defraud the federal government. However, many organizations commit fraud by requesting legitimate claims related to unintentional mistakes. These mistakes can be attributed to poor charting of the patient's course of hospitalization, providing partial treatment for complicated diseases, and longer hospital stays [8]. Reducing documentation errors not only improves reimbursements but it also improves patient safety. Thus, providers need to understand the coding process and the rules that must be followed to ensure coding objectivity and accuracy [5].

Although, the case studies described in this article are only two examples of cases that a CDI specialist provided additional oversight, similar patient care matters are resolved on a daily basis by this group of health care professionals throughout the hospital. This paper also addressed the role of a CDI specialist serving as a liaison between documentation and care. These 
specialists are able to request additional information from physicians regarding questions or issues with treatment modalities and make recommendations because they are able to review the patient's chart during their hospitalization [11,12]. Additionally, knowledge and experience of the CDI specialists may increase coding accuracy and reimbursement along with patient safety and overall outcomes. Medical billing and coding professionals are still needed to help physicians accurately code data sets post patient discharge, however, CDI specialists may be vital to patient care as they provide additional oversight in real-time.

\section{Conclusion}

CDI specialists are vital to improving data integrity and documentation in hospital settings because physicians need additional oversight for precise coding. These specialist review medical records for inconsistencies between diagnoses and treatments concurrently and make recommendations to ensure that patient care is congruent with evidence-based practice. Nurses may be best suited for this role because they have an educational background that includes an array of sciences and clinical experience in various medical specialty areas. This combination of education and experience allow nurses as CDI specialists to understand the narrative notes provided by physicians and guarantee that it's congruent with the patient's course of hospitalization.

\section{References}

1. Himmelstein DU, Woolhandler S (2016) The current and projected taxpayer shares of US health costs Am J Public Health 106: 449-52.

2. The Centers for Medicare and Medicaid Services (CMS) and the National Center for Health Statistics (NCHS) FY2018, ICD-10-CM Official Guidelines for Coding and Reporting Retrieved 2016.

3. WHO (2016) International statistical classification of disease and related health problems, $10^{\text {th }}$ revision volume 2 (instruction manual), $5^{\text {th }}$ edition.

4. DeVault K (2017) The role of coding and documentation in the quality payment program. J AHIMA 88: 52-5.

5. Carr K (2017) Best practices for HIM professionals managing CDI programs. J AHIMA 19-21.

6. Hailes J (2012) Truly Understanding Clinical Documentation Improvement for ICD-10. U.S. Indian Health Service. Retrieved August 2017

7. Lakin JR, Isaacs E, Sullivan E, Harris HA, McMahan RD, et al. (2015) Emergency Physicians' Experience with Advance Care Planning Documentation in the Electronic Medical Record: Useful, Needed, and Elusive. J Palliat Med 19: 632-8.

8. Bilyeu P, Eastes R (2013) Use of the electronic medical record for trauma resuscitation: How does this impact documentation completeness? J Trauma Nurs 20: 166-8.

9. D’Huyvetter C, Lang AM, Heimer DM, Cogbill (2014) Efficiencies gained by using electronic medical record reports in trauma documentation. J Trauma Nurs 21: 68-71.

10. Brown LR (2013) The secret life of a clinical documentation improvement specialist. Nursing 43: 10-2.

11. Arrowood D, Bailey-Woods L, Easterling S, Endicott M, Love T, et al. (2015) Best practices in the art and science of clinical documentation improvement. J AHIMA 86: 46-50.

12. Barnette E, Edincott M, Ericson C, Wieczorek M (2017) Evolving roles in clinical documentation improvement: Physician practice opportunities. J AHIMA 88: 54-8.

13. Denton DB, Endicott M, Ericson C, Love T, McDonald L, et al. (2016) Clinical validation: The next level of CDI. J AHIMA 87: 54-7.

14. The Agency for Healthcare Research and Quality (AHRQ) (2011) Toolkit for Using the AHRQ Quality Indicators How to Improve Hospital Quality and Safety retrieved 2016

15. Singer A, Kroeker AL, Yakubovich S, Duarte R, Dufault B, et al. (2016) Data quality in electronic medical records in Manitoba. CanFam Physician 63:382-9.

16. The United States Department of Justice: Office of Public Affairs, 2016 Justice News-National healthcare fraud take down, retrieved 2017. 